\title{
Anti-HER2 ADC DS-8201a
}

National Cancer Institute

\section{Source}

National Cancer Institute. Anti-HER2 ADC DS-8201a. NCI Thesaurus. Code C128799.

An antibody-drug conjug ate (ADC) composed of a monoclonal antibody targeting human epidermal growth factor receptor 2 (ERBB2; EGFR2; HER2) conjug ated to a derivative of the camptothecin analog exatecan (DXd; DX-8951 derivative), a DNA topoisomerase 1 (topoisomerase I; Top1) inhibitor, with potential antineoplastic activity. Upon administration of anti-HER2 ADC conjugate DS-8201a, the antibody moiety targets and binds to HER2 on tumor cells. Upon antibody/antigen binding and internalization, the DX8951 derivative moiety binds to and inhibits Top1-DNA complexes, which results in an inhibition of DNA replication, cell cycle arrest and tumor cell apoptosis. HER2, a tyrosine kinase receptor, is overexpressed by many cancer cell types. In addition, DS-8201a induces antibody-dependent cell-mediated cytotoxicity (ADCC) and causes a bystander killing effect, thereby killing neighboring HER2-expressing tumor cells. 